\title{
Acoustic radiation patterns of the silo music phenomenon
}

\author{
J.R. Hernández-Juárez ${ }^{1, *}, D . A$. Serrano $^{2, * *}, A$. López-Villa $^{1}$, and $A$. Medina $^{1}$ \\ ${ }^{1}$ ESIME Azcapotzalco, Instituto Politécnico Nacional, Av. de las Granjas 682, Col. Sta. Catarina, Azcapotzalco 02250 CDMX. \\ ${ }^{2}$ Instituto Tecnológico de Querétaro, Av. Tecnológico s/n, Centro, 76000 Santiago de Querétaro, Qro.
}

\begin{abstract}
In this work, we apply the theory of radiation and reception of acoustic waves in the analysis of the acoustic contributions of the silo music phenomenon. The length portion of the silo where the silo music phenomenon occurs is considered a continuous line source. The continuous line source modeled as a simple acoustic source permits the analysis of the acoustic far field, interpreting the polar graphics of the beam patterns of the acoustic contributions. We propose to apply this technique in the silo design to diminish adverse acoustic effects during the discharge process of granular materials. Additionally, we present calculations of the sound pressure level of the silo music phenomenon.
\end{abstract}

\section{Introduction}

One of the most interesting topics in acoustics is the survey of radiation and reception of acoustic waves. This theory takes the pulsating sphere as a prototype of a simple source that is useful to model the continuous line sources (CLS). The theory of radiation/reception of acoustic waves and the principle of acoustic reciprocity permits to analyze the CLS as a distribution of point sources.

On the other hand, the most accepted theory of generation of silo music phenomenon (SMP) is the slip-stick motion [1, 2]. However, almost null attention has been put on the study of the near/far acoustic field generated by the silo music phenomenon. The results of this work search to open an additional treatment of the SMP with the aim to contribute in the design of silos to reduce the annoying acoustic effects. The content of this paper is treated in the following sections. In Section 2, we give the analysis of the silo music phenomenon as a continuous line source. A hypothetical example of the behavior of the far acoustic field generated by the silo music is considered in Section 3. Section 4 is devoted to show some experimental results of the beam patterns generated during the silo music phenomenon. Finally, in Section 5 we give our conclusions.

\section{The silo music phenomenon as continuous line source}

In this work we focus our attention in the description of the acoustic far field and the radiation of the acoustic pressure due to the silo music phenomenon. Our analysis of the silo as a CLS is established as follows.

The silo can be considered as a finite line in the $y$-axis of length $\mathrm{L}$ with a diameter $\mathrm{D}$ and radius $\mathrm{r}=\mathrm{D} / 2$. The

\footnotetext{
*e-mail: jhernandezj@ipn.mx

**e-mail: arman2390@ @otmail.com

A video is available at https://doi.org/10.48448/tv6t-q656
}

granular material begins to flow with a mass flow $\dot{m}$ and discharge velocity $\mathrm{U}_{\mathrm{d}}$ through the bottom outlet of the silo with diameter $\mathrm{D}_{\text {out }}$, the SMP appears in a region of the silo denoted as $\mathrm{H}_{\mathrm{sm}}$. The region $\mathrm{H}_{\mathrm{sm}}$, follows the necessary condition $\mathrm{H}_{\mathrm{sm}}<\mathrm{L}$. $\mathrm{H}_{\mathrm{sm}}$ can be thought of as a CLS if the silo is sufficiently narrow according to the condition $\mathrm{r} \ll \mathrm{L}$. The surface of the CLS vibrates radially with a complex speed $\chi=V_{0} e^{i \omega t}$. Where $\omega=2 \pi \mathrm{f}$ is the radial frequency and $\mathrm{f}$ is the vibration frequency of the CLS in $\mathrm{Hz}$, and it is related to the wave number $\mathrm{k}$ as $\omega=\mathrm{kc}_{0}$. $\mathrm{c}_{0}$ is the sound speed in air with a value of $343 \mathrm{~m} / \mathrm{s}$. To express the acoustic far field, we choose an arbitrary point $P$ at a distance $\delta \gg \mathrm{H}_{\mathrm{sm}}$. $\mathrm{H}_{\mathrm{sm}}$ can be considered as a cylinder conformed by a series of differential elements $d y$ (see Fig. 1), every element vibrates with a differential strength element

$$
d \Xi=\frac{V_{0}}{(2 \pi r)^{-1}} d y
$$

The element $d \Xi$ produces a contribution to the acoustic pressure at $P$, defined by the differential element

$$
d P=\frac{i \rho_{0} \omega V_{0} \mathrm{r} d y e^{i\left(\omega t-\mathrm{k} \delta^{\prime}\right)}}{2 \delta^{\prime}},
$$

where $\delta^{\prime}$ is the distance from $d y$ to the point $P, i=\sqrt{-1}$, and $\rho_{0}$ is the density in air with a value of $1.23 \mathrm{~kg} / \mathrm{m}^{3}$. $\delta^{\prime}$ can be approximated to $\delta$ according to $\delta^{\prime} \approx \delta-y \sin \alpha$. After the change of $\delta^{\prime}$ by $\delta-y \sin \alpha$ and some algebraic treatment, the total contribution of the acoustic pressure at $P$ can be found by evaluating [3]

$$
P(\delta, \varphi, t)=\frac{\mathrm{ikr} \rho_{0} \mathrm{c}_{0} \mathrm{~V}_{0} \mathrm{e}^{\mathrm{i}(\mathrm{wt}-\mathrm{kr})}}{2 \delta} \int_{-\mathrm{H}_{\mathrm{sm}} / 2}^{\mathrm{H}_{\mathrm{sm}} / 2} \mathrm{e}^{\mathrm{iky} \sin \mathrm{y}} d y .
$$

An accepted solution of (2) for the description of the acoustic far field $([4,5])$ due to a CLS is given by 


$$
P(\delta, \alpha)=\wp_{y}(\delta) \Lambda(\alpha)
$$

where

$$
\wp_{y}(\delta)=\frac{1}{2} \rho_{0} \mathrm{c}_{0} V_{0}\left(\frac{\mathrm{r}}{\delta}\right) \mathrm{kH}_{\mathrm{sm}}
$$

and

$$
\Lambda(\alpha)=|\operatorname{Sinc}(\sigma)|, \sigma=\frac{1}{2} \mathrm{kH}_{\mathrm{sm}} \sin (\alpha) .
$$

$\Lambda(\alpha)$ is the directivity function and describes the acoustic radiation of $\mathrm{H}_{\mathrm{sm}}$ in the far field.

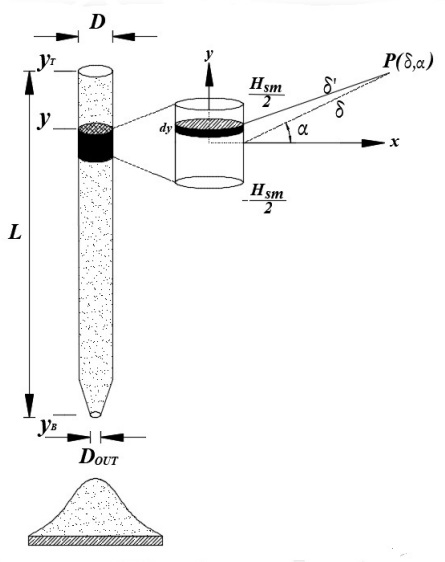

Figure 1. The silo as a continuous line source.

\section{Beam patterns of the silo music}

In this section we present a numerical example of the beam patterns generated by the silo music phenomenon. Suppose a tall and narrow silo of length $\mathrm{L}=1.5 \mathrm{~m}$ with a diameter $\mathrm{D}=0.05 \mathrm{~m}$. The silo is filled with a monodispersive granular material with a diameter $\mathrm{d}_{g}$. To assure the continuous $\dot{m}$ through the bottom silo outlet with diameter $D_{\text {out }}$, the condition $\mathrm{D}_{\text {out }}>6 \mathrm{~d}_{g}$ must be imposed. During the silo discharge process the SMP is present. For this example four discharge processes have been supposed giving different length values of $\mathrm{H}_{\mathrm{sm}}$. Conveniently starting at the same height of the silo. Taking the length $\mathrm{L}$ from the bottom to the top of the silo in the positive $y$-axis, being the bottom $\mathrm{y}_{\mathrm{B}}=0 \mathrm{~m}$ and the top $\mathrm{y}_{\mathrm{T}}$ $=1.5 \mathrm{~m}$. The silo music occurs after the grain material has been continuosly displaced $0.1 \mathrm{~m}$ from the top to the bottom of the silo. The $\mathrm{H}_{\mathrm{sm}}$ region begins at $\mathrm{y}=1.4$ $\mathrm{m}$ from the bottom and has a minimum value of length according to $\mathrm{H}_{\mathrm{sm}} \geq \lambda$, where $\lambda$ is the wavelength defined as $\lambda=c_{0} / f$. The sound emitted by the SMP possesses a rich tone, this means a great number of harmonics, a fundamental frequency and the lowest component in frequency with the biggest $\lambda$ that is related with the minimum length of $\mathrm{H}_{\mathrm{sm}}$ and the condition mentioned above. For this example, we take the lowest frequency of the SMP as $\mathrm{f}=2000 \mathrm{~Hz}$, conveniently the same for all discharges. The value of $\mathrm{f}$ gives an approximate minimum length value for $\mathrm{H}_{\mathrm{sm}} \approx 0.175 \mathrm{~m}$. The beam patterns of Fig. 3 and Fig. 4 are obtained applying (4).

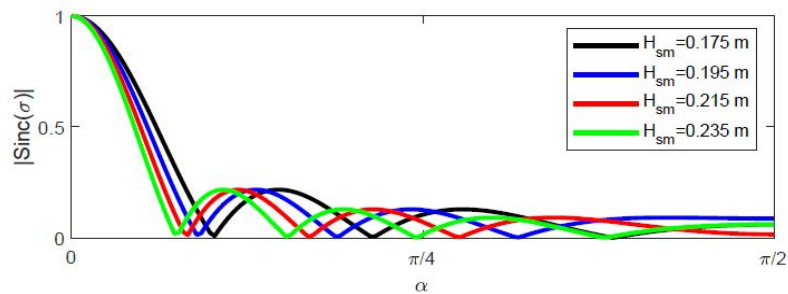

Figure 2. General behaviour of the CLS associated with different lengths of $\mathbf{H}_{s m}$. Cartesian representation of $|\operatorname{Sinc}(\sigma)|$.

For the case of the CLS, the $\operatorname{Sinc}(\sigma)$ function in Cartesian representation describes the directivity of $\mathrm{H}_{\mathrm{sm}}$. In Fig. 2 the behavior of $\operatorname{Sinc}(\sigma)$ is observed according to the length of $\mathrm{H}_{\mathrm{sm}}$, when $\mathrm{H}_{\mathrm{sm}}$ increases the radiated acoustic energy is redistributed from the main lobe to the side lobes. This effect can be better visualized in polar plots of $\Lambda(\alpha)$.
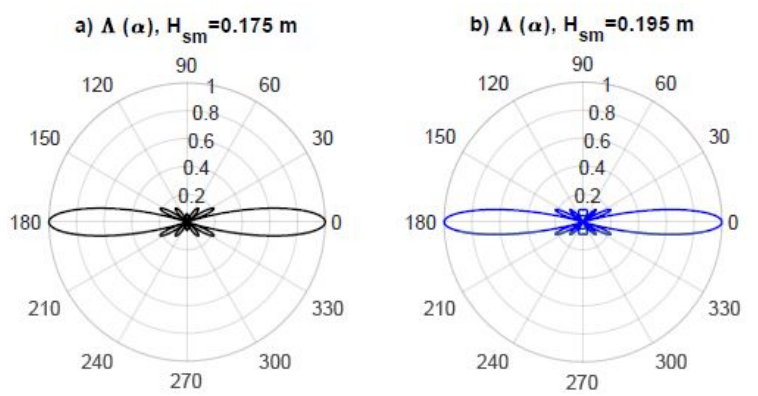

Figure 3. Polar plots of the beam patterns of the silo music phenomenon for a) $\mathrm{H}_{s m}=0.175 \mathrm{~m}$ and b) $\mathrm{H}_{s m}=0.195 \mathrm{~m}$.
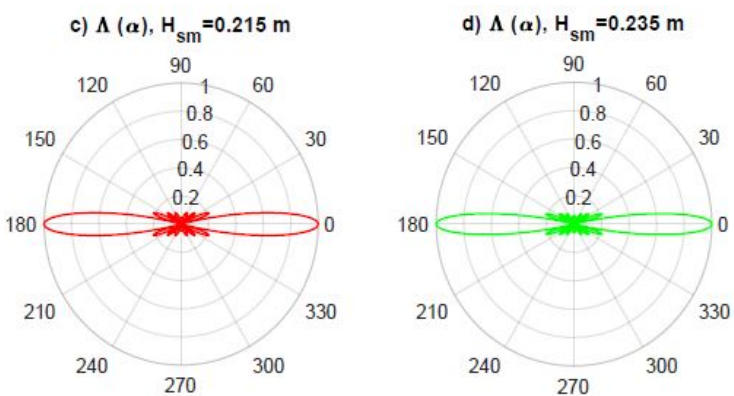

Figure 4. Polar plots of the beam patterns of the silo music phenomenon for a) $\mathrm{H}_{s m}=0.215 \mathrm{~m}$ and b) $\mathrm{H}_{s m}=0.235 \mathrm{~m}$.

From polar plots of Fig. 3 and Fig. 4 we observe the beam patterns of the acoustic energy generated by the SMP, is through out all the length $\mathrm{H}_{\mathrm{sm}}$. Most of the concentration of acoustic energy of the SMP is present in the main lobe, the directivity of CLS is guaranteed for $\mathrm{H}_{\mathrm{sm}} \approx \lambda$. As a consequence, for the same $\mathrm{f}$ when $\mathrm{H}_{\mathrm{sm}}$ increases the main lobe gets narrow and side lobes 
appear. The acoustic energy is redistributed in these side lobes. As a single conclusion, the directivity can be controlled changing the length of $\mathrm{H}_{\mathrm{sm}}$ or controlling the lowest frequency $f$ of the SMP.

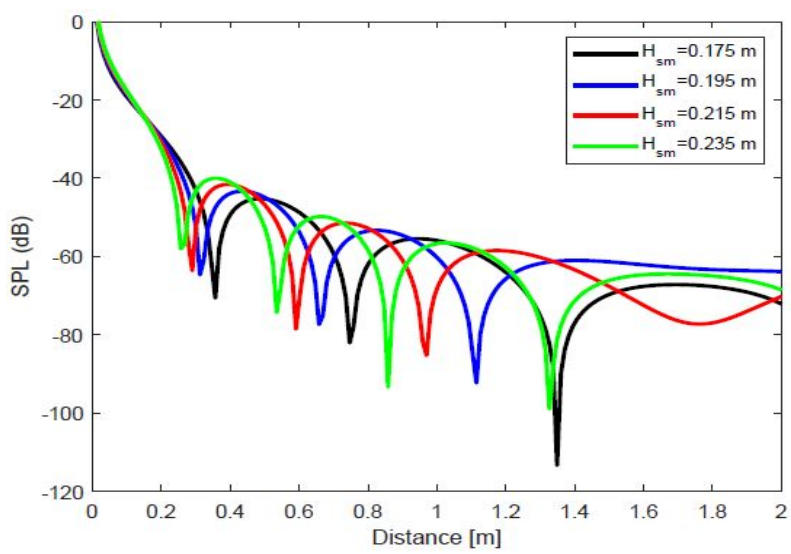

Figure 5. Evolution of the sound pressure level in $\mathrm{dB}$ of the SMP.

The sound pressure level can be obtained applying $\mathrm{SPL}=20 \log P(\delta, \alpha)$. As the observer moves away from the $\mathrm{H}_{\mathrm{sm}}$, the acoustic field drops its intensity. In Fig 5 the behavior of the acoustic field for different values of $\mathrm{H}_{\mathrm{sm}}$ can be observed, corresponding to the SMP presence during the silo discharge process. The plot in Fig. 5 was obtained considering that the observation point is located at $\delta=7 \mathrm{~m}$, according to

$$
\delta \frac{\lambda}{2}=\frac{\mathrm{L}^{2}}{4}-\frac{\lambda^{2}}{16} .
$$

Expression (5) arises from the generalization of the Pythagorean theorem applied to the CLS analysis. The surface vibration speed of the $\mathrm{H}_{s m}$ was set as $V_{0}=1 \times 10^{-5}$ $\mathrm{m} / \mathrm{s}$.

\section{Experimental application}

For this experimental example, we implement a glass tube as a silo with a length $\mathrm{L}=46.5 \mathrm{~cm}$, a diameter $\mathrm{D}=4.5 \mathrm{~cm}$. The bottom circular outlet of the silo has a diameter $\mathrm{D}_{\text {out }}$ $=0.8 \mathrm{~cm}$. The granular material used in the experiment was granulated sugar whose mean diameter $\mathrm{d}_{g}=0.95 \mathrm{~mm}$ and bulk density $\rho_{B}=1.06 \mathrm{~g} / \mathrm{cm}^{3}$ were measured in the laboratory. The silo was placed in vertical position and the bottom outlet blocked, and then the silo was filled with granular material from the open top. The continuous discharge process begins at the moment when the outlet is unlocked. The mass flow $\dot{m}$ is given by

$$
\dot{m}=\rho_{B} g^{1 / 2} \mathrm{D}_{\text {out }}^{5 / 2},
$$

where $g$ is the gravity acceleration. The discharge velocity $\mathrm{U}_{\mathrm{d}}(\dot{m})$ is related to the mass flow as

$$
\mathrm{U}_{\mathrm{d}}(\dot{m})=\frac{\dot{m}}{\rho_{B} \mathrm{~A}}
$$

where $\mathrm{A}$ is the area of the silo outlet [6]. The numerical values for the granulated sugar are $\dot{m}=19.00 \mathrm{~g} / \mathrm{s}$ and $\mathrm{U}_{\mathrm{d}}(\dot{m})=35.69 \mathrm{~cm} / \mathrm{s}$. The experiment was conducted inside an acoustic isolation chamber with the aim to avoid and reduce external noises and ground vibrations, as depicted in Fig. 6. During the discharge process of the granulated sugar the acoustic contributions were present. The sound sensor was used to capture the acoustic contributions of the SMP and the data acquisition system and Signal Processing Toolbox ${ }^{\mathrm{TM}}$ of Matlab 2020 to interpret the signals of the phenomenon in time domain and frequency domain. The total duration time measured of the silo discharge process was $t=75.02 \mathrm{~s}$. The SMP can be identified in the time domain signal according to the abrupt change of amplitude located in $t_{1}=1.8 \mathrm{~s}$ and $t_{2}$ $=11.4 \mathrm{~s}$, indicating that the region of the SMP is located near to the top of the silo. The total time of the SMP is given by $t_{s m}=t_{2}-t_{1}=9.6 \mathrm{~s}$. Knowing the length $\mathrm{L}$ and the times of the SMP, the heights where the SMP begins $\mathrm{H}_{1}$ and ends $\mathrm{H}_{2}$ are identified by applying

$$
\mathrm{H}_{\mathrm{j}}=\eta \mathrm{U}_{\mathrm{d}} \Delta t, \Delta t=t-t_{j}, \mathrm{j}=1,2
$$

where $\eta=1 / 60$ is an empirical constant value. So, for $\mathrm{H}_{1}=43.55 \mathrm{~cm}$ and $\mathrm{H}_{2}=37.84 \mathrm{~cm}$. As consequence $\mathrm{H}_{\mathrm{sm}}=\mathrm{H}_{1}-\mathrm{H}_{2}=5.71 \mathrm{~cm}$. This result indicates that the CLS associated with the silo must have a minimum approximated length of $0.0571 \mathrm{~m}$ and the frequency associated with this length must be at least $\geq 6007 \mathrm{~Hz}$, according to $\mathrm{H}_{\mathrm{sm}} \geq \lambda$.

The experimental analysis slightly differs from the idealized case presented in Section 3. This is due to the fact that the silo music experiment is an acoustic phenomenon of rich tone. In the idealized case, the choice of the lowest frequency component is arbitrary and easy, it is just necessary to follow the condition $\mathrm{H}_{\mathrm{sm}} \geq \lambda$. However, for this experimental example we can not choose the lowest component due to the fact it would be in the inferior limit of the frequency range giving a $\lambda>>\mathrm{L}$.

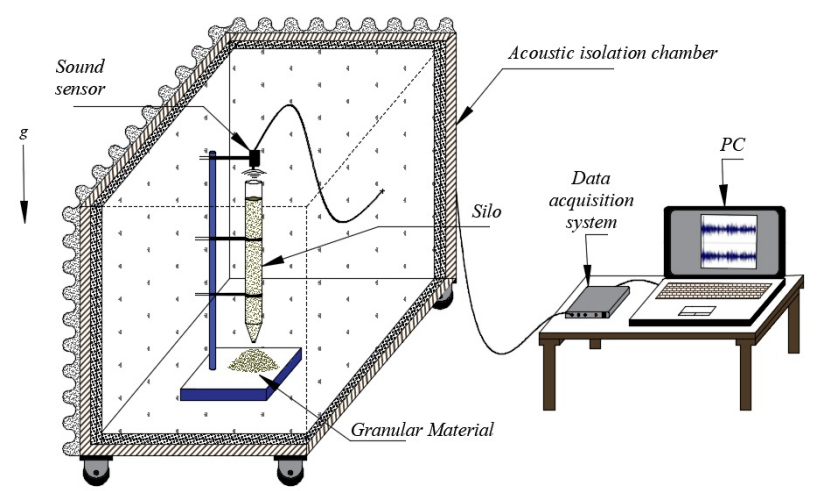

Figure 6. Disposition scheme of the experiment.

The signal obtained by the data acquisition system during the discharge process of the silo is shown in Fig. 7. Specifically, Fig. 7a is the representation of all sound contributions of the silo discharge process, including the discharge noise (low amplitude signal) and acoustic 
contributions of the silo music phenomenon. The silo music phenomenon is indicated by the perpendicular lines (square ends) to $t$-axis. In Fig. 7b, the amplification of the SMP is shown. The frequency components associated to the SMP of Fig. 7b are observed in Fig. 7c. According to the analysis, the best frequency selection must be for $\mathrm{f} \geq 6007 \mathrm{~Hz}$. From Fig. 7c, the fundamental frequency is given by $f_{d}=6875 \mathrm{~Hz}$, and a good selection for a low frequency component is $\mathrm{f}_{\mathrm{LC}}=6406 \mathrm{~Hz}$, giving $\lambda=0.0535$ $\mathrm{m}$ fulfilling the condition $\mathrm{H}_{\mathrm{sm}} \geq \lambda$. Now, we have the capability to perform the analysis of the acoustic far field of the SMP of the experiment. Applying (3) and (4), the beam pattern and sound pressure level are present in the plots of Fig. 8 y Fig. 9.
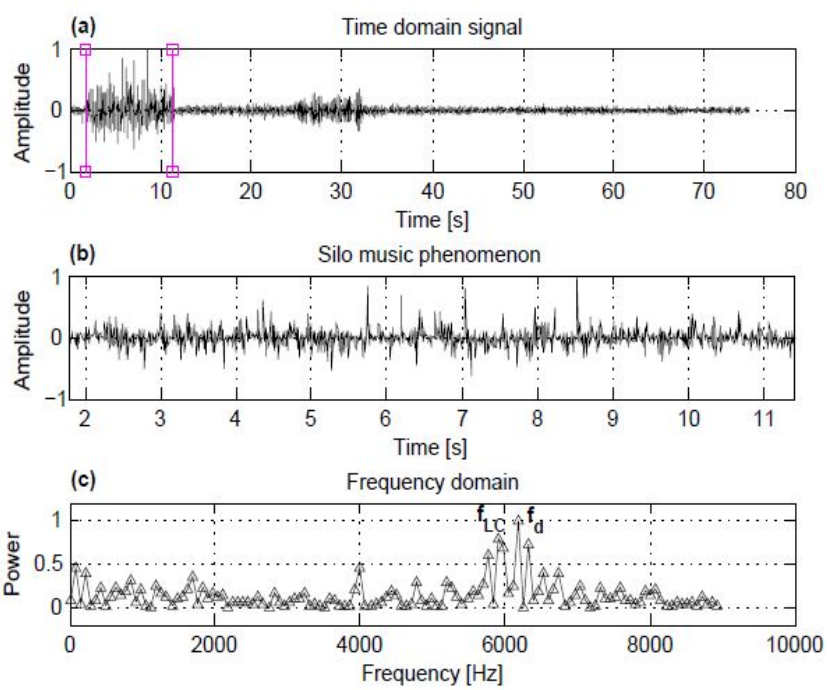

Figure 7. Signal of the SMP in time domain and frequency domain.

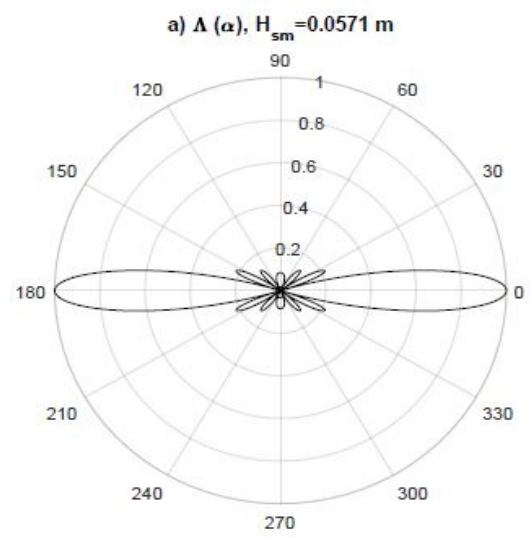

Figure 8. Beam pattern of the experimental SMP.

In Fig. 8 we observe the beam pattern generated during the experimental SMP where the most acoustic energy is concentrated in the main lobe and some portion of the total energy is distributed in the side lobes. The plot in Fig. 9 shows the behavior of the sound pressure level. The sound pressure level was calculated considering a far field distance $\delta=2 \mathrm{~m}$ from the source according to (5).

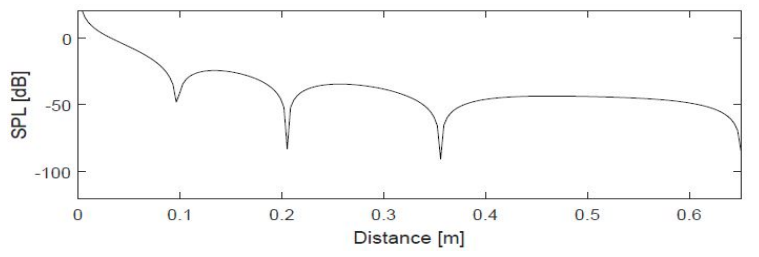

Figure 9. Sound pressure level of the experimental SMP.

\section{Conclusion and observations}

This work presents a methodology to study the behavior of the radiated acoustic energy due to the acoustic contributions generated by the SMP. The beam patterns generated by the directivity function permit to know the distribution of the acoustic energy generated by the phenomenon. We propose this methodology as application to reduce the undesirable acoustic effects of the SMP by reducing the lobes. The presence of the SMP is unpredictable due to the nature and properties of the granular materials in conjunction with the silo properties. It is well known that the SMP occurs in the upper section of the silo near to the top. This fact can be used in the design of silos to modify mechanically the silo properties and manipulate the $\mathrm{H}_{\mathrm{sm}}$ region to diminish or avoid the SMP. Finally, the rich tone of the SMP does not make the treatment of the analysis easy, however as we have shown the signal processing theory is a useful tool in the analysis of the signals in time domain and frequency domain generated by the SMP. In future works the silo wall thickness will be considered.

Acknowledgements.- JRHJ acknowledges CONACYT for the SNI fellowship. Authors wish to thank the IPN, especially the SEPI ESIME Azcapotzalco for providing the facilities to conduct the experiment in its installations.

\section{References}

[1] J. Y. Ooi, J. Chavez-Sagarnaga, Z. Zhong, D. M. Campbell, J. M. Buick, C. A. Greated, J. Eng. Mech. 131, 299 (2005)

[2] J. M. Buick, J. Y. Ooi, J. Chavez-Sagarnaga, A. Pearce, G. Houghton, J. Phys. D: Appl. Phys. 37, 2751 (2004)

[3] T. D. Rossing, Springer Handbook of acoustics, Springer, USA C.A., 2014

[4] D. R. Dowling, Proc. Mtgs. Acout. 19, 025002 (2013)

[5] J. Jackson, Classical electrodynamics, John Wiley and Sons, 1999

[6] D. A. Serrano, F. Sánchez-Silva, J. Klapp, P. Tamayo, A. Medina, G. Domínguez, Recent Advances in Fluid Dynamics with Environmental Applications, Ed. J. Klapp, Springer, 2016 